\title{
Avaliação da resistência ao desgaste erosivo e abrasivo de revestimentos WC 12 Co aplicados por aspersão térmica HVOF
}

\author{
Evaluation of erosive and abrasive wear \\ resistance of the WC 12 Co coatings \\ applied HVOF thermal spray
}

\author{
Maria Júlia Xavier Belém ${ }^{1}$, Carlos Roberto Camello Lima ${ }^{1}$, \\ Amanda Kuhl ${ }^{1}$, Flávio Camargo ${ }^{2}$
}

\footnotetext{
${ }^{1}$ Programa de Pós Graduação em Engenharia de Produção - PPGEP/UNIMEP, 13.451-900, Santa Bárbara D’Oeste, SP, Brasil.

e-mail: mjuliaxb@gmail.com, carlos.lima1@unimep.br, amandakuh195@gmail.com

${ }^{2}$ Ogramac, 13.830-000, Santo Antônio de Posse, SP, Brasil.

e-mail: flavio@ogramac.com.br
}

\section{RESUMO}

Revestimentos são aplicados com o intuito de prolongar a vida útil de componentes que operam em ambientes agressivos. Revestimentos de material metal-cerâmico, conhecidos como cermet, como o WC-Co, consistem de carbonetos de tungstênio imersos numa matriz de cobalto e são utilizados como materiais resistentes ao desgaste. O processo de aspersão HVOF (High Velocity Oxygen Fuel) é o mais adequado quando se quer obter revestimentos de carbonetos. A aspersão térmica HVOF apresenta revestimentos de excelente qualidade com relação à porosidade, dureza, resistência, densidade, rugosidade e aderência ao substrato. O presente estudo investiga e compara a resistência ao desgaste erosivo e abrasivo de revestimentos obtidos por HVOF, aplicados sobre substratos de aço AISI 1020. Foram realizados de ensaios de erosão, utilizando um tribômetro específico, teste de abrasão seguindo a norma ASTM G65-16, análise por micrografia eletrônica de varredura (MEV), microdureza e rugosidade dos revestimentos. Os materiais utilizados foram as ligas WC $12 \mathrm{Co.}$ Como resultado do ensaio de desgaste erosivo, o revestimento mostrou-se como uma boa opção quando se trata de resistência em ângulos de incidência menores. Na primeira hora de ensaio, a de taxa de erosão foi $42 \%$ maior em ângulo de $90^{\circ}$ do que em ângulo de impacto de $30^{\circ}$. No ensaio de desgaste abrasivo, o resultado do desgaste volumétrico pode ser observado nas micrografias após ensaio, notando que houve uma retirada de carbonetos da superfície do revestimento, resultando em desgaste, na qual, as amostras tiveram uma perda volumétrica de 2,68 $\pm 0,28 \mathrm{~mm}^{3}$. Tais resultados indicam o material WC 12Co uma boa opção quando se trata de resistência ao desgaste para proteção de componentes mecânicos industriais.

Palavras-chave: revestimentos cermets, WC 12Co, HVOF, desgaste abrasivo, desgaste erosivo.

\begin{abstract}
Coatings are applied in order to extend the life of components operating in harsh environments. Metalceramic coatings known as cermet, such as WC-Co, consist of tungsten carbides immersed in a cobalt matrix and are used as wear-resistant materials. The HVOF (High Velocity Oxygen Fuel) spraying process is most suitable when one wants to obtain carbide coatings. The HVOF thermal spray presents excellent quality coatings with respect to porosity, hardness, strength, density, roughness and adhesion to the substrate. The present study investigates and compares the erosive and abrasive wear resistance of coatings obtained by HVOF, applied on AISI 1020 steel substrates. Were performed erosion tests using a specific tribometer, abrasion test according to ASTM G65-16, scanning electron microscope (SEM), microhardness and roughness of the coatings. The materials used were WC $12 \mathrm{Co}$ alloys. As a result of the erosive wear test, the coating has proved to be a good choice when dealing with resistance at lower angles of incidence. In the first hour of testing, erosion rate was $42 \%$ higher at $90^{\circ}$ angle than at $30^{\circ}$ impact angle. In the abrasive wear test, the result of the
\end{abstract}


volumetric wear can be observed in the micrographs after the test, noting that there was a removal of carbides from the surface of the coating, resulting in wear, in which the samples had a volumetric loss of $2.68 \pm 0.28$ $\mathrm{mm}^{3}$. Such results indicate the WC $12 \mathrm{Co}$ material a good choice when it comes to wear resistance for protection of industrial mechanical components that suffer wear.

Keywords: cermets coatings, WC $12 \mathrm{Co}, \mathrm{HVOF}$, abrasive wear, erosive wear.

\section{INTRODUÇÃO}

O desgaste é uma das principais causas que provocam a degradação dos componentes de engenharia em indústrias como petroquímica, energia e aeroespacial [1]. O desgaste pode ocorrer em diversas formas, como por fricção, deslizamento, impacto, abrasão, erosão e outras condições de serviço. É um dano progressivo, envolvendo, muitas vezes, grande perda de material [2].

Segundo WANG et al. [3], a erosão e a abrasão são consideradas as principais causas de falhas de componentes e equipamentos industriais. A erosão é causada, principalmente, pela colisão de partículas de deslizamento e pela ação de combustíveis sólidos, líquidos e gasosos, sobre a superfície do componente em serviço, atuando como fonte de deterioração de componentes mecânicos de motores de aeronaves, equipamentos de processamento químico, entre outros [4]. A abrasão ocorre quando partículas duras ou protuberâncias deslizam ou são forçadas a se moverem contra e ao longo de uma superfície sólida, provocando a remoção ou degradação da superfície do material [5].

Uma forma de diminuir ou mesmo prevenir a degradação provocada por o fenômeno de desgaste é a aplicação de revestimentos nas superfícies dos componentes a serem protegidos. Revestimentos são aplicados a fim de prolongar a vida útil de componentes/sistemas que operam em ambientes agressivos. Em diversos projetos de engenharia, usar um material estruturalmente adequado e um revestimento que possa proteger este material de situações com alta agressividade do ambiente de aplicação mostra-se uma técnica economicamente viável [6].

A aplicação dos revestimentos pode ser feita por técnicas como deposição física de vapor (PVD), deposição química de vapor (CVD), soldagem, cromo duro e, especialmente, aspersão térmica [7].

A aspersão térmica é um grupo de processos nos quais materiais metálicos ou não metálicos são aplicados em uma condição fundida ou semifundida sobre um substrato preparado, formando um revestimento [8]. No processo, existem diversas variáveis como: velocidade e distância de aspersão; taxa de material; fluxo de combustível; entre outros, os quais podem ser modificados dependendo do material e processo de aspersão, a fim de obter revestimentos que atendam a finalidade imposta. $\mathrm{O}$ material de revestimento pode estar na forma de pó, nano ou micro estruturado, vareta, cordão ou arame. A ligação entre o revestimento e o substrato pode ser mecânica, metalúrgica, química, física ou uma combinação destas formas [9]. Diferentes materiais podem ser aplicados por esse processo, como os cermets.

Revestimentos cermets são usados em diversos ambientes industriais [10], que requerem resistência ao desgaste, como em componentes aeroespaciais e indústria petroquímica [11].

A utilização de revestimentos cermets aplicados por aspersão térmica apresenta alta resistência ao desgaste abrasivo [12], sem prejudicar o desempenho do componente revestido. LI e YANG [13] afirma que grande parte da resistência ao desgaste dos cermets como o WC-Co é devido a sua alta dureza e tenacidade.

Segundo LI e YANG [13], entre os processos de aspersão térmica para obtenção dos revestimentos cermets, como o carboneto de tungstênio com cobalto (WC-Co), o D-Gun foi muito utilizado na década de 50, tendo muita aplicação na indústria aeronáutica por gerar revestimentos densos e com excelente resistência adesiva, porém com a baixa disponibilidade do sistema, foi substituído pelo plasma e, posteriormente, pelo HVOF, que apresenta revestimentos com características similares aos aplicados por D-Gun.

O HVOF (High Velocity Oxygen Fuel), considerado umas das principais técnicas de aspersão térmica [14,15], sendo reconhecido pelo depósito de revestimentos com baixa porosidade, alta adesão e alta resistência ao desgaste $[16,17]$ tendo como características alta velocidade de impacto e, comparado com o plasma, uma baixa temperatura que resulta em menor decomposição do carboneto de tungstênio durante o processo de aspersão [10]. Altos níveis de WC retidos indicam um revestimento com maior dureza e tenacidade e boa resistência ao desgaste [18].

O processo HVOF utiliza equipamento que trabalha a alta pressão de gases, alcançando velocidades supersônicas, resultando em maiores densidades de revestimento, melhor aderência e estruturas de revestimento mais uniformemente dispersas devido a grandes deformações de partículas que ocorrem no momento do impacto. O revestimento apresenta porosidades inferiores a 1\%, e uma microdureza de 40-68 HRC. Se- 
gundo LIU et al. [19], HVOF é ideal para a deposição de revestimentos cermet, como a liga WC-Co, resistente ao fenômeno de desgaste.

Dada à importância da engenharia de superfícies e suas possibilidades, este estudo teve como objetivo a avaliação de revestimentos tipo cermets, especificamente o carboneto de tungstênio com cobalto (WC $12 \mathrm{Co}$ ) aplicados pelo processo de aspersão térmica HVOF. Foram realizados ensaios de desgaste abrasivo e erosivo e a avaliação da resistência a erosão e abrasão do revestimento WC 12 Co se deu por meio da perda de massa obtida após os testes.

\section{MATERIAIS E MÉTODOS}

Para obtenção das amostras revestidas, foi utilizado como substrato o aço AISI 1020. O material utilizado como revestimentos sobre o substrato foi a liga WC $12 \mathrm{Co}$, na forma de pó, aplicada por aspersão térmica HVOF/JP-5000. Devido a sua alta dureza, esse tipo de material, chamados de cermet, é altamente resistente ao desgaste [20]. O carboneto de tungstênio (WC), ligado a materiais metálicos como o Co, apresenta excelente combinação de resistência ao desgaste e propriedades mecânicas em alta temperatura [12].

A preparação do substrato AISI 1020 e aplicação dos revestimentos foram realizadas com equipamentos da empresa Ogramac. Posteriormente, foram feitas medidas de microdureza, medição de rugosidade e análises por microscopia eletrônica de varredura em todas as amostras. Ainda, realizaram-se ensaios de desgaste erosivo, utilizando um equipamento tribômetro específico, e desgaste abrasivo, seguindo a Norma ASTM G65-16 [21].

No processo de aspersão térmica é muito importante e indispensável preparar adequadamente o substrato, pois a limpeza e a rugosidade da superfície do substrato que garantem a adesão do revestimento. $\mathrm{O}$ substrato, chapa de aço, passou por uma limpeza de grau Sa3, com função de, além da limpeza superficial, aumentar a rugosidade da superfície da base, a fim de obter uma melhor aderência do revestimento. Segundo a Norma ISO 8501-1 [22], o grau Sa (limpeza por jateamento abrasivo) é comumente utilizado na limpeza de superfície antes da aspersão. O grau de limpeza Sa3 é o recomendado para aplicação de revestimento com o processo HVOF, na qual a superfície quando observada a olho nu deve estar isenta de óleos, graxa e sujeiras visíveis, devendo ainda apresentar uma cor metálica uniforme em toda superfície.

Após ter a rugosidade desejada, antes da aplicação dos revestimentos, o substrato foi pré-aquecido, com uma temperatura de $70^{\circ} \mathrm{C}$. O preaquecimento da superfície a ser aspergida melhora a adesão do revestimento a ser aplicado, reduzindo o stress térmico residual [23].

Para aplicação dos revestimentos, foi utilizado o equipamento de aspersão térmica HVOF de alta pressão TAFA JP-5000 (HP-HVOF), conforme parâmetros de aspersão apresentados na Tabela 1.

BERNDT e LENLING [24] afirmam que na aspersão térmica, a distância entre a pistola e o substrato pode afetar a microestrutura do revestimento final. Se a distância for consideravelmente pequena, a fusão das partículas é prejudicada; se a velocidade de impacto não for suficientemente alta, não permitirá a obtenção de camadas densas de revestimento. Neste estudo, a distância de aspersão foi mantida em torno de $380 \mathrm{~mm}$, feita manualmente pelo operador, na qual a cada 10 passes, aproximadamente, a temperatura do substrato revestido era controlada, juntamente com a espessura da camada, para não ultrapassar a espessura especificada. Por fim, foram obtidos revestimentos com espessura em torno de $500 \sim 600 \mu \mathrm{m}$.

Tabela 1: Parâmetros do processo de aspersão HVOF.

\begin{tabular}{l|l|l|l}
\hline VAZÃO DE QUEROSENE & VAZÃO DE OXIGÊNIO & TAXA DE PÓ & DISTÂNCIA DE ASPERSÃO \\
\hline $6 \mathrm{gph}$ & $2000 \mathrm{scfh}$ & $76 \mathrm{~g} / \mathrm{min}$ & $380 \mathrm{~mm}$ \\
\hline
\end{tabular}

A rugosidade dos revestimentos concluídos foram realizadas utilizando um Rugosímetro eletromecânico - Mitutoyo, Suftest - 211 .

As análises da microestrutura dos depósitos foram feitas por microscopia eletrônica de varredura (MEV), para isso as amostras foram embutidas, lixadas e polidas. Feito o processo metalográfico, também foram feitas medidas de microdureza, utilizando um microdurômetro marca Shimadzu. O ensaio foi de acordo com a norma DIN 50133 - Pirâmide de diamante Vickers - com carga de 2,9N, que corresponde a 300 g $\left(\mathrm{HV}_{0,3}\right)$, e feitas sete medições ao longo do corte transversal do revestimento.

\subsection{Teste de desgaste erosivo}


Foram utilizados quatro amostras com revestimentos WC 12 Co de dimensão $23 \mathrm{~mm}$ x $10 \mathrm{~mm}$ x 5 mm. Foram realizadas seis horas de ensaio em cada amostra utilizando um equipamento construído, que consiste em uma furadeira de bancada Schulz, modelo FSB 16P, modificada, acoplando dispositivos para utilização como um Tribômetro (Figura 1).

Tribômetro consiste em um equipamento utilizado em testes, a fim de estudar o mecanismo de desgaste, por meio da caracterização tribológica dos mais diversos materiais, envolvendo especificações como velocidade, ângulo e material erosivo, por exemplo.

Como mistura erosiva foi utilizada $300 \mathrm{~g}$ de partícula erosiva mais $700 \mathrm{~g}$ de água. As amostras ficaram posicionadas em contato com erosão por meio de um dispositivo, que possui como opção, variar o ângulo de impacto para $30^{\circ}$ ou $90^{\circ}$.
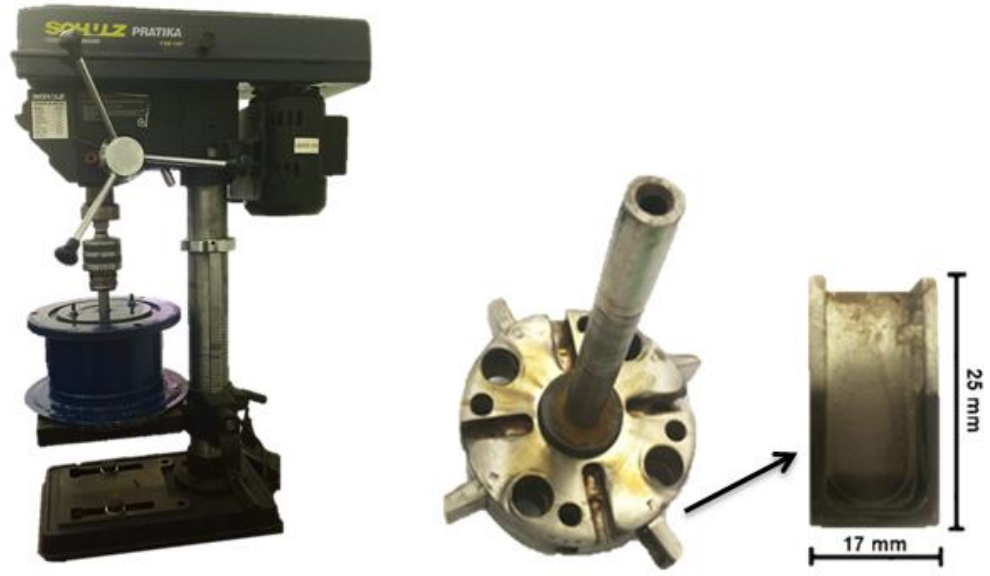

Figura 1: Furadeira modificada para funcionar como tribômetro para ensaio de desgaste erosivo, com destaque no dispositivo porta amostras.

A velocidade de impacto das partículas está diretamente relacionada com as dimensões do dispositivo de porta amostras. A Figura 2 mostra as medidas fundamentais para o cálculo da velocidade.

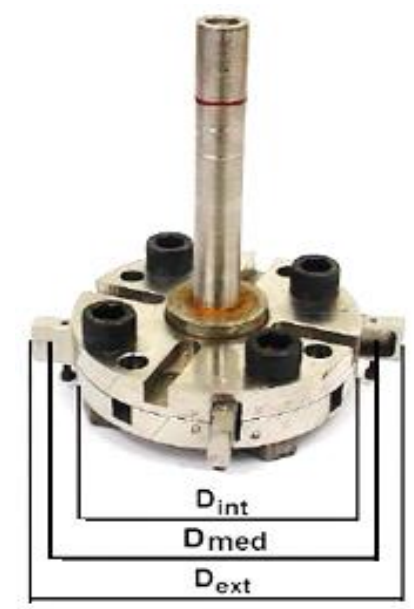

Figura 2: Dispositivo com dimensões especificadas, necessárias para o cálculo da velocidade de impacto.

A velocidade de impacto $\left(V_{\text {Impacto }}\right)$ foi calculada através da Equação 1 , onde $(n)$ representa o número de rotações por minuto do dispositivo de porta amostras.

$$
V_{\text {Impacto }}=\frac{\pi \cdot D_{\text {med }} \cdot n}{60 \times 10^{3}}
$$


O diâmetro médio ( $D_{\text {med }}$ ) foi determinado pela Equação 2.

$$
D_{\text {med }}=\frac{D_{\text {ext }}+D_{\text {int }}}{2}
$$
$\mathrm{mm})$.

Onde: $D_{\text {ext }}=$ diâmetro exterior do dispositivo $(130 \mathrm{~mm}) ; D_{\text {int }}=$ diâmetro interior do dispositivo $(100$

Portanto, feito o cálculo através da Equação 2, o valor do diâmetro médio é de $115 \mathrm{~mm}$. Para a rotação (n), foi escolhido um valor de $1550 \mathrm{rpm}$, disponível na furadeira, e calibrada através do uso de um tacômetro digital; Com os dados necessários obtidos, foi utilizada a Equação 1, e teve como resultado da velocidade de impacto das partículas erodentes de $9,33 \mathrm{~m} / \mathrm{s}$.

Para medição da taxa de desgaste, as amostras foram pesadas antes e em cada intervalo de uma hora do teste de erosão utilizando uma balança digital Shimadzu, modelo AY220, peso máximo de 220g e precisão de $0,001 \mathrm{~g}$. A calibração da balança foi feita utilizando um peso padrão de $200 \mathrm{~g}$, com uma imprecisão de 0,001g. O ensaio de desgaste erosivo foi realizado de acordo com os parâmetros apresentados na Tabela 2.

Tabela 2: Parâmetros utilizados em teste de desgaste erosivo.

\begin{tabular}{l|l}
\hline PARTÍCULA EROSIVA & Areia de quartzo $(\mathrm{SiO} 2-30 / 40)$ de granulometria de $300 / 420 \mu \mathrm{m}$ \\
\hline TEMPERATURA DO ENSAIO & $\sim 29^{\circ} \mathrm{C} ;$ \\
\hline VELOCIDADE DAS PARTÍCULAS & $9,33 \mathrm{~m} / \mathrm{s}(1550 \mathrm{rpm})$ \\
\hline ÂNGULOS DE IMPACTO & $30^{\circ}$ e $90^{\circ}$ \\
\hline
\end{tabular}

\subsection{Teste de desgaste abrasivo}

O ensaio de desgaste abrasivo foi realizado baseando-se na Norma ASTM G65-16. Refere-se a um método de teste padrão a fim de medir a abrasão usando um equipamento de roda de borracha e, como abrasivo, areia seca com tamanho de grão e composição controlados. O teste de abrasão da roda de borracha tem sido objeto de vários estudos [25, 26, 27].

Nessa pesquisa, foram utilizadas quatro amostras com dimensões $25 \mathrm{~mm}$ x $75 \mathrm{~mm}$ x 12,5 mm, para obter uma maior confiabilidade nos resultados. Para medição da taxa de desgaste, as amostras foram pesadas antes e após o teste de abrasão.

Para avaliação da resistência abrasiva do carboneto de tungstênio, foi seguido o procedimento B [21], apresentando uma carga de 130 Newtons e 2000 revoluções.

A resistência ao desgaste abrasivo das amostras foi avaliada através da variação de massa das amostras após teste e, posteriormente, normalizada para perda de volume de acordo com a Equação 3 [21].

Perda de volume $\left(\mathrm{mm}^{3}\right)=\frac{\text { Perda de massa }(\mathrm{g})}{\text { Densidade }\left(\mathrm{g} / \mathrm{cm}^{3}\right)} .1000$

Para simular o fenômeno de desgaste, o abrasivo foi inserido entre a amostra padronizada e um anel de borracha que contém dureza especificada, como mostra a Figura 3. A amostra foi pressionada com uma carga especificada pela norma, contra a roda de borracha que realiza um movimento giratório. Simultaneamente foi introduzido um fluxo de areia a uma vazão de $400 \mathrm{~g} / \mathrm{min}$, fazendo com que ocorra o fenômeno de abrasão sobre a superfície a ser analisada. 


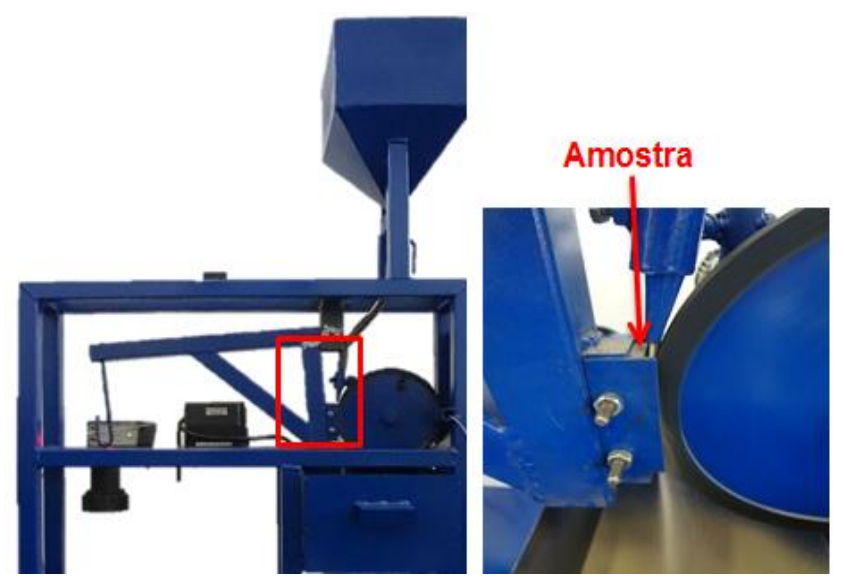

Figura 3: Equipamento utilizado em ensaio de desgaste abrasivo seguindo a norma ASTM G65-16, com destaque na amostra sofrendo abrasão durante ensaio.

\section{RESULTADOS}

Esta seção apresenta os resultados encontrados de rugosidade, ensaio de microdureza, análise por microscopia eletrônica de varredura (MEV) e ensaios de desgaste erosivo e abrasivo.

Foram feitas cinco medições de rugosidade no revestimento, determinadas à média e desvio padrão, listados na Tabela 3.

Tabela 3: Medidas da rugosidade em Ra $(\mu \mathrm{m})$ dos revestimentos WC 12Co.

\begin{tabular}{ccccccc}
\hline \multicolumn{3}{c}{ MEDIDAS } & & MÉDIA & DESVIO PADRÃO \\
\hline 5,17 & 5,95 & 5,74 & 5,47 & 5,14 & 5,49 & 0,35 \\
\hline
\end{tabular}

A Tabela 4 apresenta as medidas de microdureza dos revestimentos aplicados por HVOF.

Tabela 4: Medidas de microdureza dos revestimentos: carga de 300g.

\begin{tabular}{cccccccccc}
\hline REVESTIMENTO & \multicolumn{4}{c}{ MEDIDAS $\left(\mathbf{H V}_{\mathbf{0 . 3}}\right)$} & \multicolumn{3}{c}{ MÉDIA } & $\begin{array}{c}\text { DESVIO } \\
\text { PADRÃO }\end{array}$ \\
\hline WC 12Co & 1086 & 1095 & 1091 & 1135 & 1048 & 1104 & 1090 & 1092 & 26 \\
\hline
\end{tabular}

Para análises das micrografias, as amostras foram cortadas e sua seção transversal foi analisada por Microscopia Eletrônica de Varredura. A Figura 4 apresenta as micrografias dos revestimentos antes dos ensaios de desgaste.

Na micrografia da Figura 4a nota-se um revestimento denso com baixa porosidade. A micrografia da Fig. 4b mostra uma distribuição uniforme, com tamanhos variados dos carbonetos (fase mais clara) na matriz de cobalto. Estruturas semelhantes foram obtidas nos trabalhos de LAMANA et al. [28], DEJUN e TIANYUAN [29]. 

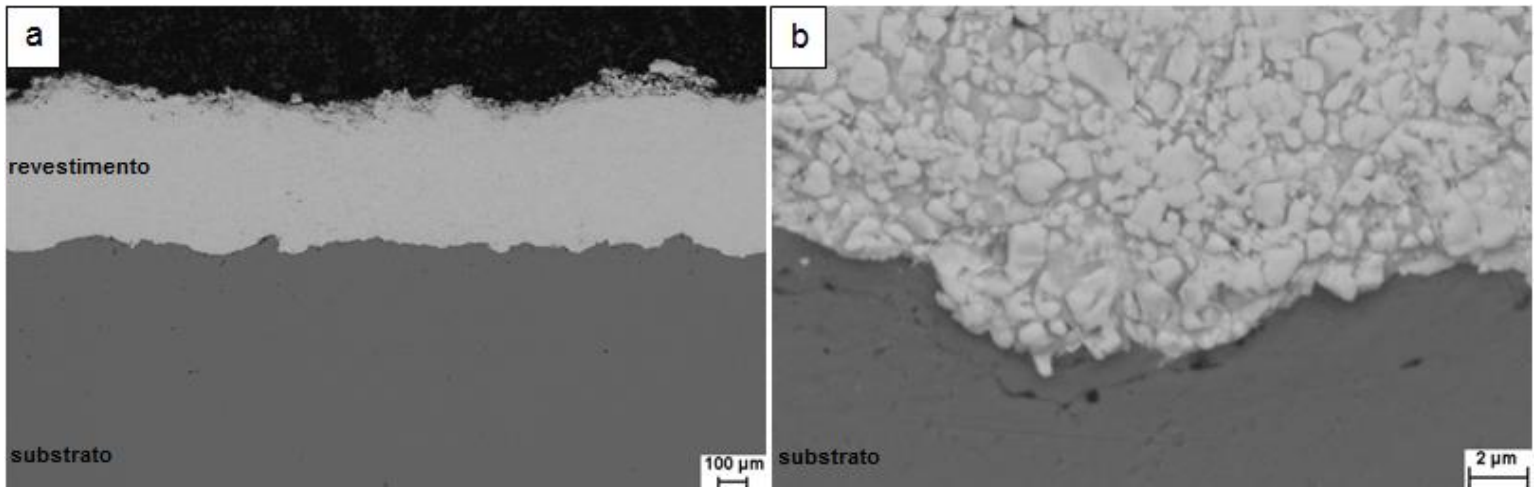

Figura 4: a) Revestimento WC $12 \mathrm{Co}$ aplicado por HVOF (100x); b) carbonetos de tamanhos variados na matriz metálica (2000x)

\subsection{Teste de desgaste erosivo}

O ensaio de desgaste erosivo foi realizado com ângulos de incidência das partículas de $30^{\circ}$ e $90^{\circ}$. A resistência ao desgaste foi avaliada pela perda de massa sofrida após o teste e depois normalizada para perda de volume (Eq. 3).

Para o material carboneto de tungstênio (WC 12Co) utilizado como revestimento, a densidade considerada foi de $14,2 \mathrm{~g} / \mathrm{cm}^{3}$ [30].

A Tabela 5 apresenta os valores de perda de massa total após 6 horas de ensaio de desgaste erosivo com ângulo de incidência de $30^{\circ}$ e $90^{\circ}$ para as amostras, e o desgaste volumétrico, que ocorreu nos revestimentos.

Tabela 5: Resultados da perda de massa do ensaio de desgaste erosivo.

\begin{tabular}{|c|c|c|c|c|c|c|}
\hline $\begin{array}{l}\text { REVESTIMENTO/ } \\
\text { ÂNGULO DE } \\
\text { INCIDÊNCIA }\end{array}$ & $\begin{array}{l}\text { PESO INI- } \\
\text { CIAL Oh (g) }\end{array}$ & $\begin{array}{l}\text { PESO APÓS } \\
6 \mathrm{~h}(\mathrm{~g})\end{array}$ & $\begin{array}{l}\text { PERDA DE } \\
\text { MASSA TO- } \\
\text { TAL (g) }\end{array}$ & $\begin{array}{l}\text { DESGASTE } \\
\text { VOLUMÉTRICO } \\
\left(\mathrm{mm}^{3}\right)\end{array}$ & $\begin{array}{l}\text { MÉDIA DO DES- } \\
\text { GASTE VOLUMÉ- } \\
\text { TRICO }\left(\mathrm{mm}^{3}\right)\end{array}$ & $\begin{array}{l}\text { DESVIO } \\
\text { PADRÃO }\end{array}$ \\
\hline \multirow{2}{*}{$\mathrm{WC} 12 \mathrm{Co} / 30^{\circ}$} & 14,901 & 14,878 & 0,023 & 1,620 & \multirow{2}{*}{1,761} & \multirow{2}{*}{0,199} \\
\hline & 14,819 & 14,792 & 0,027 & 1,901 & & \\
\hline \multirow{2}{*}{ WC $12 \mathrm{Co} / 90^{\circ}$} & 14,776 & 14,735 & 0,041 & 2,887 & \multirow{2}{*}{2,923} & \multirow{2}{*}{0,050} \\
\hline & 14,491 & 14,449 & 0,042 & 2,958 & & \\
\hline
\end{tabular}

\subsection{Teste de desgaste abrasivo}

O ensaio de erosão foi realizado seguindo o procedimento B da Norma ASTM G65-16. Foram utilizados quatro amostras para obter uma maior confiança nos resultados, com tamanhos especificados pela norma.

A resistência ao desgaste foi avaliada pela variação de massa que as amostras tiveram após o teste, normalizada para perda de volume de acordo com a Eq. 3, apresentada anteriormente. A Tabela 6 apresenta os resultados obtidos do teste de abrasão.

Tabela 6: Resultados da perda de massa do ensaio de desgaste abrasivo.

\begin{tabular}{l|l|l|l|l}
\hline $\begin{array}{l}\text { REVESTIMENTO } \\
\text { WC 12CO }\end{array}$ & PESO INICIAL Oh (g) & $\begin{array}{l}\text { PESO APÓS EN- } \\
\text { SAIO (g) }\end{array}$ & $\begin{array}{l}\text { PERDA DE MASSA } \\
\text { TOTAL (g) }\end{array}$ & $\begin{array}{l}\text { DESGASTE VOLUMÉ- } \\
\text { TRICO (mm })^{3}\end{array}$ \\
\hline CP 1 & 206,252 & 206,215 & 0,037 & 2,620 \\
\hline CP 2 & 210,521 & 210,487 & 0,034 & 2,387 \\
\hline CP 3 & 210,444 & 210,409 & 0,035 & 2,500 \\
\hline CP 4 & 205,834 & 205,791 & 0,043 & 3,028 \\
\hline Média & 208,263 & 208,226 & 0,037 & 2,634 \\
\hline
\end{tabular}




\begin{tabular}{l|l|l|l|l}
\hline Desvio Padrão & 2,569 & 2,572 & 0,004 & 0,280 \\
\hline
\end{tabular}

\section{DISCUSSÃO}

Esta seção apresenta as discussões dos resultados encontrados de rugosidade, ensaio de microdureza, análise por microscopia eletrônica de varredura (MEV) e ensaios de desgaste erosivo e abrasivo.

A rugosidade medida no revestimento foi de $5,49 \pm 0,35 \mu \mathrm{m}$, próximo ao valor encontrado por CELIK et al. [31]. Revestimentos aplicados por HVOF apresentam menor rugosidade do que outros processos de aspersão [7]. No ensaio de desgaste erosivo realizado, uma baixa rugosidade superficial dos corpos de prova pode significar uma menor perda de material, pois as partículas erodentes agiriam de maneira mais agressiva em superfícies com rugosidade alta.

A microdureza medida foi elevada, em torno de $1092 \pm 26\left(\mathrm{HV}_{0.3}\right)$, condizentes com o tipos de material que foi aplicado, próximos aos valores encontrados por KHAN et al. [32] e BRANDT [33].

KHAN et al. [32] dizem que a dureza é uma propriedade importante, capaz de indicar o desempenho de um revestimento, com possibilidade de obter informações sobre a resistência ao fenômeno de desgaste do revestimento. No estudo, os autores observaram que os revestimentos que apresentaram uma maior dureza, tiveram uma maior resistência ao desgaste.

\subsection{Teste de desgaste erosivo}

A Figura 5 apresenta graficamente os valores de perda de massa (Tabela 5) total após 6 horas de ensaio de desgaste erosivo com ângulo de incidência de $30^{\circ} \mathrm{e} 90^{\circ}$, transformados em desgaste volumétrico.

A Figura 6 apresenta graficamente o comportamento da taxa de erosão de cada amostra para os ângulos de incidência de erodente a $30^{\circ}$ e $90^{\circ}$, mostrando que os revestimentos WC $12 \mathrm{Co}$ analisados tendem a um aumento da taxa de erosão, quando submetidos a ângulo de impacto de $90^{\circ}$, semelhante ao estudo feito por SANTANA et al. [34]. No estudo, os autores avaliaram o comportamento ao desgaste erosivo de dois materiais, WC 12Co e WC 10Co 4Cr aplicados por HVOF, concluindo que o WC 10Co 4Cr apresentou uma melhor resistência ao desgaste erosivo por ser um material com maior dureza, devido a presença do cromo e por o revestimento $\mathrm{WC} 12 \mathrm{Co}$ possuir uma maior quantidade da fase frágil $\mathrm{W}_{2} \mathrm{C}$ que como consequência diminui a resistência do revestimento, do que a encontrada no revestimento WC $10 \mathrm{Co} 4 \mathrm{Cr}$.

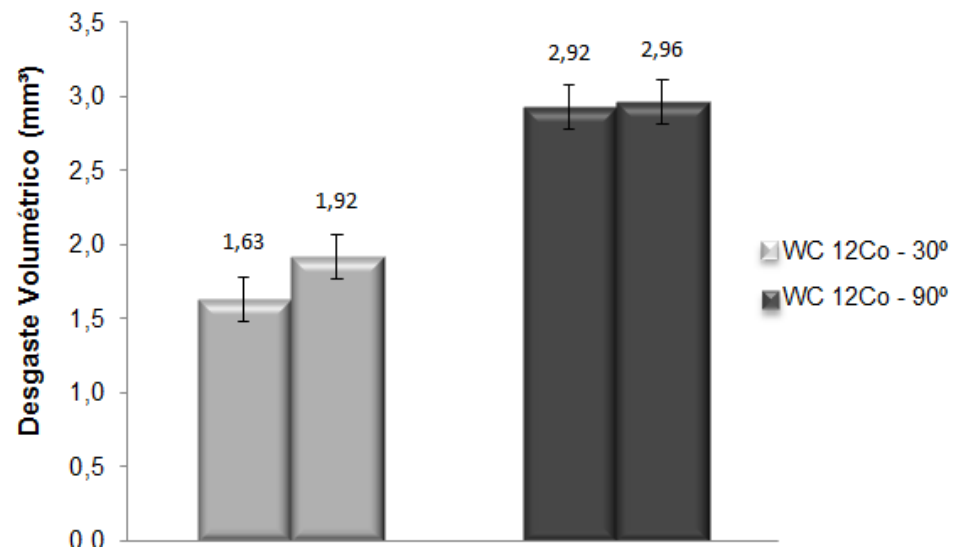

Figura 5: Desgaste volumétrico das amostras WC 12Co após teste de desgaste erosivo à ângulos de incidência $30^{\circ}$ e $90^{\circ}$.

Observando a Fig. 6, os revestimentos WC 12Co, na primeira hora de ensaio, tiveram uma de taxa de erosão de $42 \%$ maior em ângulo de $90^{\circ}$ do que em ângulo de impacto de $30^{\circ}$, podendo indicar que os revestimentos analisados possuem comportamentos de um mecanismo de desgaste erosivo quebradiço, como aponta HUSSAINOVA [35]. Ainda, o autor indica que ao comparar materiais dúcteis e quebradiços, considerando a taxa de erosão em função do ângulo de impacto, são obtidos diferentes comportamentos. Porém, segundo SANTANA et al. [34] o teor de $12 \%$ de Co presente no revestimento deveria apresentar um mecanismo de desgaste dúctil. Tal comportamento dos revestimentos analisados faz com que considere o mecanismo de desgaste erosivo como uma combinação de mecanismos dúcteis e quebradiços, em que baixos ângulos de 
impacto é mais visível um mecanismo de desgaste dúctil e em maiores ângulos um mecanismo de desgaste quebradiço.

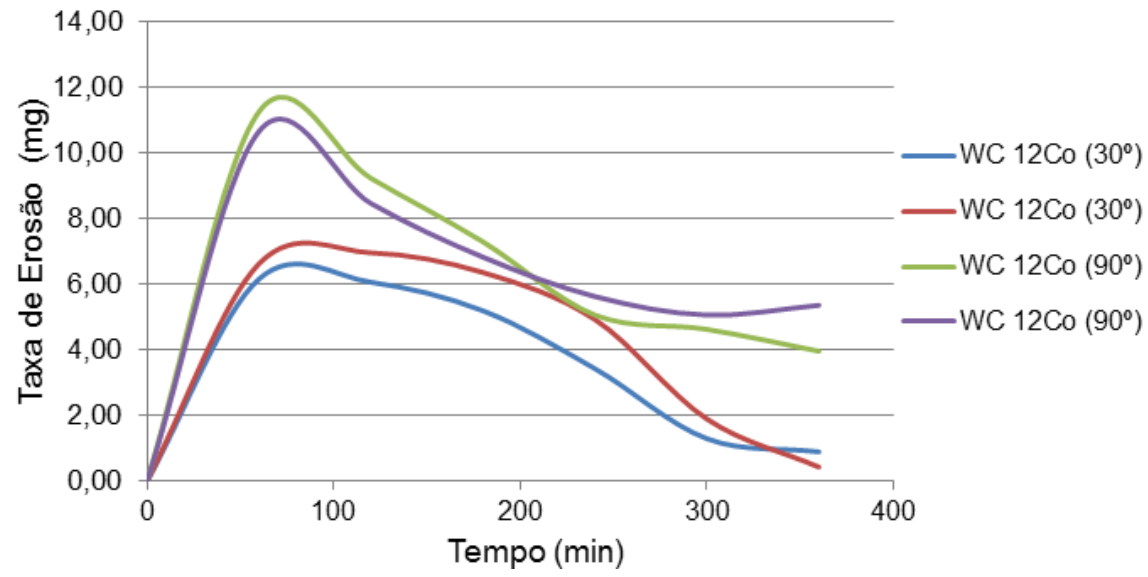

Figura 6: Taxa de erosão em miligrama percorrido para cada hora de ensaio erosivo.

O gráfico da Figura7 mostra a perda de massa das amostras em relação ao tempo. É observado que a perda de material é mais acentuada em baixo ângulo de incidência $\left(30^{\circ}\right)$, resultado semelhante ao encontrado por WANG et al. [3] que concluíram que em menores ângulos de impacto, os revestimentos WC 12Co depositados pelo processo HVOF apresentam uma melhor resistência ao desgaste erosivo do que em maiores ângulos de impacto $\left(90^{\circ}\right)$.

Devido ao revestimento WC 12 Co apresentar carbonetos na sua estrutura, em maiores ângulos de ataque há uma menor resistência ao desgaste, provocando uma maior perda de massa do que em menores ângulos, muito característicos de materiais cerâmicos. Em baixos ângulos os materiais cerâmicos são suficientes para reduzir o desgaste erosivo devido à ausência de deformação plástica da sua estrutura.

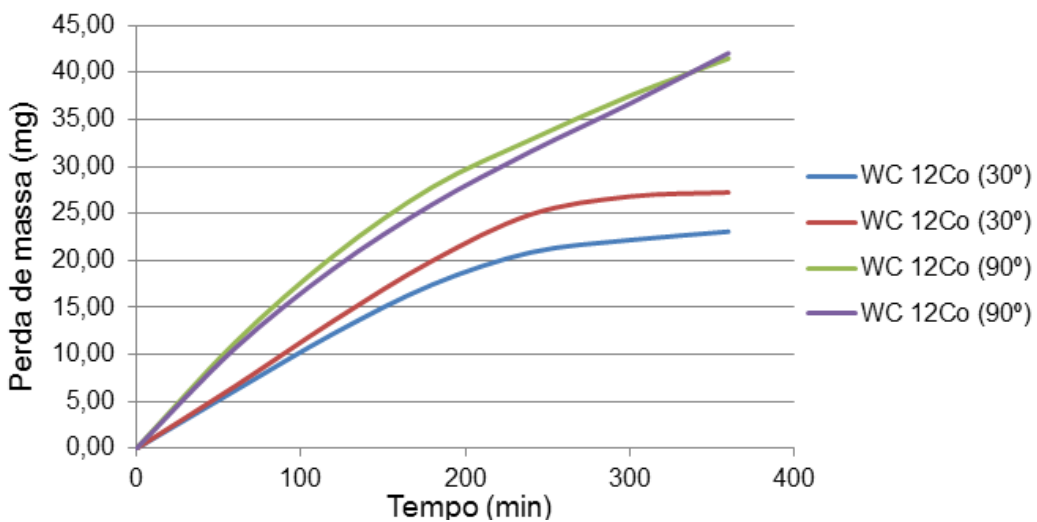

Figura 7: Perda de massa das amostras em relação a cada hora de ensaio erosivo.

O comportamento da velocidade com que ocorre o desgaste pode ser observado no gráfico da Figura 8. A velocidade de desgaste aumenta com o tempo, para ambos os corpos de prova às máximas são encontradas no início do ensaio, onde as superfícies das amostras apresentam maior rugosidade, e as mínimas velocidades estão próximas a 6 horas de ensaio, após isso os revestimentos tiveram perda de material, deixando a superfície mais lisa, com menor rugosidade do que no início do deste, com isso os desgastes dos revestimentos começam a ser mínimos. 


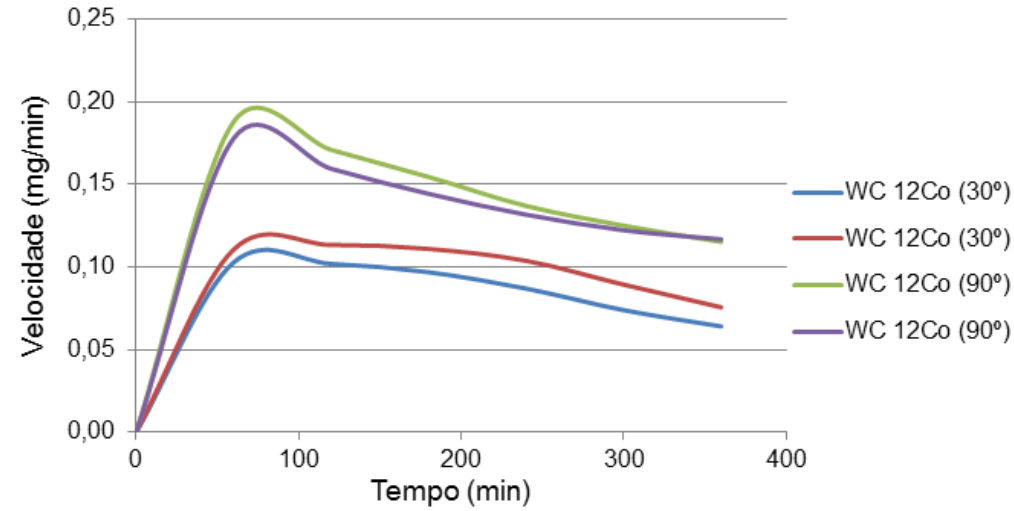

Figura 8: Velocidade de desgaste das amostras em relação a cada hora de ensaio erosivo.

\subsection{Teste de desgaste abrasivo}

A resistência ao desgaste foi avaliada por meio dos resultados de desgaste volumétrico sofrido pelos revestimentos das quatro amostras, como apresenta o gráfico da Figura 9. O desgaste volumétrico do material WC12Co no ensaio de abrasão realizado foi de $2,63 \pm 0,28 \mathrm{~mm}^{3}$.

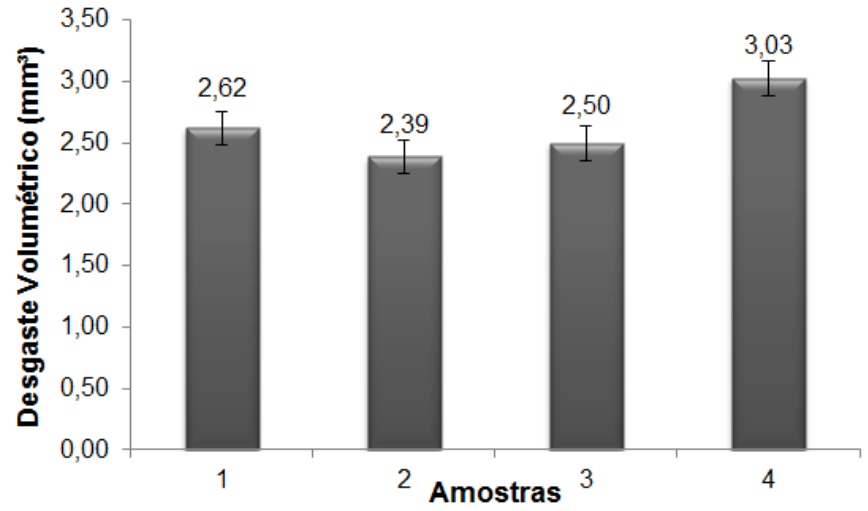

Figura 9: Desgaste volumétrico das amostras WC 12Co após teste de desgaste abrasivo.

Em um estudo feito por BARBEZAT et al. [36] foi analisado o comportamento ao desgaste abrasivo de revestimentos WC com $12 \%$ e $17 \%$ de Co, e com outros materiais como Ni e CoCr, aplicados pelos processos Continous Detonation Spraying (CDS), Detonation Gun Spraying (DGS), Atmospheric Plasma Spraying (APS) e Vacum Plasma Spraying (VPS). Na pesquisa, realizaram testes de abrasão de roda de borracha, com areia de quartzo, e observaram que os revestimentos WC 12Co aplicados por CDS e DGS apresentaram melhor resistência ao desgaste por a estrutura conter um baixo nível de fases frágeis de $\mathrm{W}_{2} \mathrm{C}$, em comparação com os outros revestimentos analisados. O estudo aponta que o Co presente no revestimento é uma vantagem quando se trata de resistência à abrasão, como pode ser observado nos resultados obtidos nesse estudo, apontando a ligação WC-Co como uma opção para proteção de ambientes onde se encontra abrasão.

O revestimento WC 12 Co também foi estudado por LIMA et al. [37]. Os autores realizaram o mesmo teste de abrasão de roda de borracha, entretanto o revestimento foi aplicado por o processo de aspersão flame spray. Como resultado, as amostras tiveram um maior desgaste volumétrico, cerca de $62,6 \%$ a mais, do que o revestimento desse estudo, aplicado por HVOF, em condições de teste similares.

O HVOF é o processo ideal para aplicação de cermets, apresentando revestimentos mais densos, menor rugosidade e porosidade, e com melhor resistência à abrasão, como pode ser observado em LEGOUX et al. [38]. No estudo, os autores analisaram revestimentos $\mathrm{WC} 10 \mathrm{Co} 4 \mathrm{Cr}$ aplicados por quatro tipos de aspersão térmica de alta velocidade, sendo que três foram HVOF (JP-5000, JP-5000ST e DJ-2700) e um plasma. Foi observado que a aspersão por HVOF/JP-5000 apresentou maiores velocidades e menores temperaturas das partículas durante a aplicação dos revestimentos e uma menor porosidade dos depósitos. E como resultado da resistência ao desgaste abrasivo de acordo com a norma ASTM G65, os revestimentos aplicados por 
HVOF/JP-5000 apresentaram maior resistência à abrasão do que os aspergidos pelos outros métodos de aspersão analisados.

A microestrutura do revestimento foi analisada após ensaio de abrasão por MEV (Figura 10), sendo possível visualizar o desgaste superficial sofrido pelas amostras. Pode ser observado que com a abrasão, houve o deslocamento dos carbonetos (região mais clara) na matriz de cobalto, deixando partes do revestimento sem carbonetos (região escura), e aparecimento de microfissuras originadas pelo sentido do desgaste.

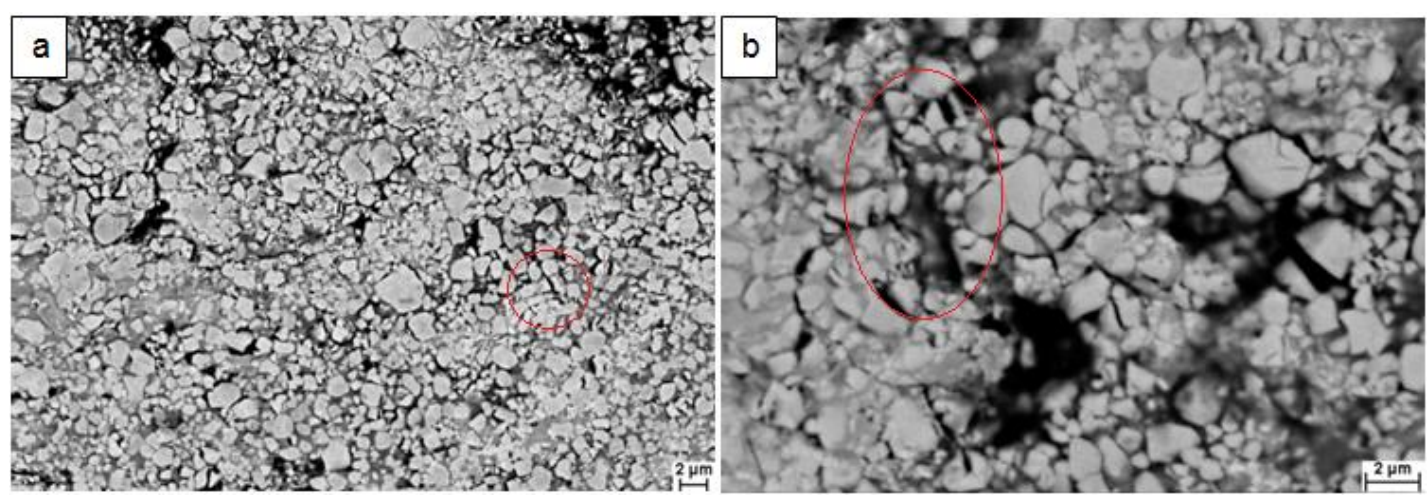

Figura 10: a) Superfície das amostras após ensaio de desgaste abrasivo (5000x); b) deslocamento de carbonetos presentes na matriz de cobalto, destacando microfissura (10000x).

\section{CONCLUSÕES}

O material WC $12 \mathrm{Co}$, aplicado pelo processo de aspersão térmica HVOF, é uma boa opção quando se trata de resistência ao desgaste, resultando em revestimentos densos, com baixa rugosidade e alta dureza.

No ensaio de desgaste erosivo, pode ser visto que o mecanismo de desgaste está diretamente associado ao ângulo de incidência das partículas de erosão e às propriedades mecânicas dos materiais envolvidos. $\mathrm{O}$ estudo mostrou que em ângulos de incidência das partículas próximos a $90^{\circ}$, as amostras apresentaram menor resistência à erosão, comportamento esse explicado devido ao material ser considerado um cermet, possuindo características semelhantes à de materiais cerâmicos, muito utilizados para proteção de componentes mecânicos contra degradação.

No ensaio de abrasão foi observado que os revestimentos cerâmicos nesse estudo tiveram uma maior resistência ao desgaste abrasivo do que outros revestimentos identificados na literatura como mostram os resultados de desgaste volumétrico, apresentando uma menor taxa de abrasão. As micrografias das superfícies das amostras apresentadas na Fig. 10 apontaram o efeito causado pela abrasão, em que com o deslocamento dos carbonetos, a superfície fica mais vulnerável a sofrer desgaste.

\section{AGRADECIMENTOS}

À Ogramac, empresa parceira, por fornecer as amostras utilizadas neste trabalho.

O presente trabalho foi realizado com apoio da Coordenação de Aperfeiçoamento de Pessoal de Nível Superior - Brasil (CAPES) - Código de Financiamento 001.

\section{BIBLIOGRAFIA}

[1] ZAFAR, S., SHARMA, A. K., “Abrasive and erosive wear behaviour of nanometric WC-12Co microwave clads", Wear, v. 346-347, pp. 29-45, 2016.

[2] TAN, J. C., LOONEY, L., HASHMI, M. S. J., "Component repair using HVOF thermal spraying”, Journal of Materials Processing Technology, v. 92-93, pp. 203-208, 1999.

[3] WANG, Q., CHEN, Z. H., DING, Z. X., et al., "Performance study of abrasive wear and erosive wear of WC-12Co coatings sprayed by HVOF", In: Nanoelectronics Conference. INEC 2nd IEEE International. IEEE, 2008. pp. 340-344, 2008.

[4] THAKUR, L., ARORA, N., "A comparative study on slurry and dry erosion behaviour of HVOF sprayed WC-CoCr coatings", Wear, v. 303, n. 1-2, pp. 405-411, 2013. 
[5] STACHOWIAK, G.W., BATCHELOR, A. W., Engineering Tribology. 2 ed., Great Britain, ButterworthHeinemann, 2001.

[6] LIU, Z., CABRERO, J., NIANG, S., et al., "Improving corrosion and wear performance of HVOFsprayed Inconel 625 and WC-Inconel 625 coatings by high power diode laser treatments", Surface and Coatings Technology, v. 201, 2007.

[7] AL-MUTAIRI, S., HASHMI, M. S. J., YILBAS, B. S., et al., "Microstructural characterization of HVOF/plasma thermal spray of micro/nano WC-12\%Co powders", Surface and Coatings Technology, v. 264, pp. 175-186, 2015.

[8] PAWLOWSKI, L., The science and engineering of thermal spray coatings, John Wiley \& Sons, 2008.

[9] LIMA, C.R.C., TREVISAN, R.E., Aspersão Térmica: Fundamentos e Aplicações, 2 ed., São Paulo, Artliber, 2007.

[10] YANG, Q., SENDA, T., OHMORI, A., "Effect of carbide grain size on microstructure and sliding wear behavior of HVOF-sprayed WC-12\% Co coatings", Wear, v. 254, n. 1-2, pp. 23-34, 2003.

[11] SAHA, G. C., MAHMUD, T. A., KHAN, T. I. "Development of novel "Duplex NiCr-coated" nanostructured HVOF WC-17Ni(80/20)Cr coatings for severe wear applications", IN: ASME 2013 International Mechanical Engineering Congress and Exposition. American Society of Mechanical Engineers, pp. 16, 2013.

[12] LIAO, H., NORMAND, B., CODDET, C., "Influence of coating microstructure on the abrasive wear resistance of WC/Co cermet coatings", Surface and Coatings Technology, v. 124, n. 2-3, pp. 235-242, 2000.

[13] LI, C. J., YANG, G. J., "Relationships between feedstock structure, particle parameter, coating deposition, microstructure and properties for thermally sprayed conventional and nanostructured WC-Co", International Journal of Refractory Metals and Hard Materials, v. 39, pp. 2-17, 2013.

[14] AK, N. F., TEKMEN, C., OZDEMIR, I., et al., "NiCr coatings on stainless steel by HVOF technique", Surface and Coatings Technology, v. 173-174, pp. 1070-1073, 2003.

[15] STOKES, J., LOONEY, L., "HVOF system definition to maximise the thickness of formed components", Surface and Coatings Technology, v. 148, n. 1, pp. 18-24, 2001.

[16] JACOBS, L., HYLAND, M. M., DE BONTE, M., "Comparative Study of WC-Cermet Coatings Sprayed via the HVOF and the HVAF Process", Journal of Thermal Spray Technology, v. 7, n. 2, pp. 213 218,1998

[17] MATEEN, A., SAHA, G. C., KHAN, T. I., et al., "Tribological behaviour of HVOF sprayed nearnanostructured and microstructured WC-17wt.\%Co coatings", Surface and Coatings Technology, v. 206, n. 6, pp. 1077-1084, 2011.

[18] SÁNCHEZ, E., BANNIER, E., SALVADOR, M. D., et al., "Microstructure and wear behavior of conventional and nanostructured plasma-sprayed WC-Co coatings", Journal of thermal spray technology, v. 19, n. 5, pp. 964-974, 2010.

[19] LIU, W. H., SHIEU, F. S., HSIAO, W. T., "Enhancement of wear and corrosion resistance of iron-based hard coatings deposited by high-velocity oxygen fuel (HVOF) thermal spraying", Surface and Coatings Technology, v. 249, pp. 24-41, 2014.

[20] XIE, Y., JIANG, J. J., TUFA, K. Y., et al., "Wear resistance of materials used for slurry transport", Wear, v. 332-333, pp. 1104-1110, 2015.

[21] ASTM: American Society for Testing and Materials. "Standard Test Method for Measuring Abrasion Using the Dry Sand/Rubber Wheel Apparatus”, ASTM G65-16, 2016.

[22] ISO 8501-1:1998, "Preparation of Steel Substrates before Application of Paints and Related Products Visual Assessment of Surface Clealiness", 1988.

[23] PAREDES, R. S. C., AMICO, S. C., D’OLIVEIRA, A. S. C. M., "The effect of roughness and preheating of the substrate on the morphology of aluminium coatings deposited by thermal spraying", Surface and Coatings Technology, v. 200, n. 9, pp. 3049-3055, 2006.

[24] BERNDT, C.C., LENLING, W.J., Handbook of Thermal Spray Technology. New York, ASM International, 2004.

[25] STEVENSON, A. N. J., HUTCHINGS, I. M., "Development of the dry sand/rubber wheel abrasion test", Wear, v. 195, n. 1, pp. 232-240, 1996. 
[26] CHACÓN-NAVA, J. G., MARTÍNEZ-VILLAFAÑE, A., ALMERAYA-CALDERON, F., et al., "Some remarks on particle size effects on the abrasion of a range of Fe based alloys", Tribology International, v. 43, n. 8, pp. 1307-1317, 2010 .

[27] THAKARE, M. R., WHARTON, J. A., WOOD, R. J. K., et al., Effect of abrasive particle size and the influence of microstructure on the wear mechanisms in wear-resistant materials. Wear, v. 276-277, p. 16-28, 2012.

[28] LAMANA, M. S., PUKASIEWICZ, A. G. M., SAMPATH, S., "Influence of cobalt content and HVOF deposition process on the cavitation erosion resistance of WC-Co coatings", Wear, v. 398-399, n. December 2017, pp. 209-219, 2018.

[29] DEJUN, K., TIANYUAN, S., "Wear behaviors of HVOF sprayed WC-12Co coatings by laser remelting under lubricated condition", Optics and Laser Technology, v. 89, n. September 2016, pp. 86-91, 2017.

[30] BERGER, L. M., VUORISTO, P., MANTYLA, T., et al., "Microstructure and properties of WC-Co-Cr coatings", In: Thermal Spray: practical solutions for engineering problems, ASM International, Ohio, EUA, pp. 97-106, 1996.

[31] CELIK, E., CULHA, O., UYULGAN, B., et al., "Assessment of microstructural and mechanical properties of HVOF sprayed WC-based cermet coatings for a roller cylinder", Surface and Coatings Technology, v. 200, n. 14-15, pp. 4320-4328, 2006.

[32] KHAN, T. I., SAHA, G., GLENESK, L. B., "Nanostructured composite coatings for oil sand's applications", Surface Engineering, v. 26, n. 7, pp. 540-545, 2010.

[33] BRANDT, O. C., "Mechanical properties of HVOF coatings", Journal of Thermal Spray Technology, v. 4, n. 2, pp. 147-152, 1995.

[34] SANTANA, Y. Y., LA BARBE'RA-SOSA, J. G., BENCOMO, A., et al., "Influence of mechanical properties of tungsten carbide-cobalt thermal spray coatings on their solid particle erosion behavior", Surface Engineering, v. 28, n. 4, pp. 237-243, 2012.

[35] HUSSAINOVA, I., "Microstructure and erosive wear in ceramic-based composites". Wear, v. 258, n. 14, pp. 357-365, 2005.

[36] BARBEZAT, G., NICOL, A. R., SICKINGER, A., "Abrasion, erosion and scuffing resistance of carbide and oxide ceramic thermal sprayed coatings for different applications", Wear, v. 162-164, n. PART A, pp. 529-537, 1993.

[37] LIMA, C. R. C., LIBARDI, R., CAMARGO, F., et al., "Assessment of abrasive wear of nanostructured WC-Co and Fe-based coatings applied by HP-hVOF, flame, and wire arc spray", Journal of thermal spray technology, v. 23, n. 7, pp. 1097-1104, 2014.

[38] LEGOUX, J. G., ARSENAULT, B., BOUYER, V., et al., "Evaluation of four high velocity thermal spray guns using WC-10\% Co-4\% Cr cermets", Journal of Thermal Spray Technology, v. 11, n. 1, pp. 86-94, 2002.

\section{ORCID}

Maria Júlia Xavier Belém

Carlos Roberto Camello Lima

Amanda Kühl

Flavio Camargo https://orcid.org/0000-0002-1602-8898

https://orcid.org/0000-0002-7669-4059

https://orcid.org/0000-0001-5259-2958

https://orcid.org/0000-0001-8007-7005 